\title{
Effects of Atrial Fibrillation on the Coronary Flow at Different Heart Rates: a Computational Approach
}

\author{
C Gallo, L Ridolfi, S Scarsoglio \\ Politecnico di Torino, Torino, Italy
}

\begin{abstract}
Atrial fibrillation $(A F)$ has several effects on the cardiovascular system responses. This study focuses on the consequences of $A F$ on the coronary blood flow, by exploiting a computational approach. 2000 heartbeat periods (RR) were simulated for 5 different mean heart rates $(H R)$, ranging from 50 to $130 \mathrm{bpm}$. The resulting flow rate signals at the coronary level were analysed through a specific set of hemodynamic parameters. Three main results emerge during AF: (i) maximal coronary flow rates modify with $H R$, (ii) the coronary perfusion begins to be impaired when exceeding 90-110 bpm, and (iii) the coronary perfusion pressure is not a good estimate of the coronary blood flow at HRs higher than 90-110 bpm.
\end{abstract}

\section{Introduction}

AF is the most widespread cardiac arrhythmia, with an estimated number of 14-17 million patients in 2030 in Europe only [6]. AF mainly affects elderly subjects, is responsible for serious heart-related problems such as heart failure and stroke, and leads to an increase in cardiovascular morbidity and mortality.

AF is able to induce a number of changes in the standard hemodynamic parameters which are of great medical interest. To have a clear understanding of the AF-induced alterations, it is essential to determine if AF alone can promote extra cardiovascular disorders, irregardless of potential concomitant pathologies which typically arise with $\mathrm{AF}$ (valvular disorders, hypertension, atrial dilatation, etc...).

To this aim, the present work focuses on the relation between AF and coronary flow alterations. It is well known that, even in absence of coronary artery disease (CAD), abnormalities of myocardial perfusion (and perfusion reserve) are possible in AF patients, who often experience symptoms of myocardial iscahemia [2]. However, the variations in the physiological flows at coronary level due to $\mathrm{AF}$ have been poorly investigated so far and a complete view on this topic is still lacking.

Therefore, the effects of AF on the coronary flow in hu- mans are here analysed. This is done considering the fibrillated flow rate signals along the left anterior descending (LAD) region for five different mean heart rates.

\section{Methods}

The present study uses the physically-based multi-scale (OD and 1D) mathematical model proposed by Guala et al. [1,5]. This model includes left heart, with mitral and aortic valves, 48 large-medium arteries, 24 arterial bifurcations and 18 microcirculation groups. The right heart, the venous return and the cerebral circulation are neglected.

The left coronary circulation model incorporates 8 large-medium arteries, which are described through the 1D mass and momentuum balance equations, as the arterial tree vessels. Each coronary artery is closed by a specific 0D microvascular model, which describes three transmural layers: the subepicardium, the midwall and the subendocardium. All the layers are forced by an intramyocardial pressure and are divided in two compartments: the arterial one and the venous one. The generic compartment is represented by a resistance and a capacitance, with the addiction of an intermediate resistance between the arterial and venous sides. While capacitances remain constant in time, resistances are functions of time. This choice allows us to adequately simulate the autoregulation capacity, assuring the expected coronary blood flow supply. The coronary OD microvascular model adopted in this work is the one proposed by Mynard et al. [3].

To simulate AF at a given mean HR, we imposed an artificially-built stochastic sequence of RRs, following the procedure described by Scarsoglio et al. [4]. The in silico RR beating reproduces what clinically observed: the RR series in AF exhibits a reduced temporal correlation and an increased temporal variability, with respect to the normal sinus rhythm (NSR).

In this work, $2000 \mathrm{RRs}$ were simulated in $\mathrm{AF}$ conditions for five different mean cardiac frequencies: 50-70-90-110$130 \mathrm{bpm}$. Therefore, 2000 fibrillated pressure and flow rate signals were obtained for each simulated HR over the whole model domain. Since the goal of this work is to explore the coronary flow behaviour in $\mathrm{AF}$, the focus is on 
the flow rate signals at a LAD section.

\section{Hemodynamic parameters}

In order to identify the changes in the coronary flow rate during AF, key quantities of the LAD flow rate signal were calculated for the $2000 \mathrm{RRs}$ at all the cardiac frequencies of interest. A representation of some of these quantities is provided in Fig.1, which shows a typical LAD flow rate signal, $Q_{L A D}(t)$, during a single heartbeat period.

We considered the minimal flow rate $\left(Q_{m i n}\right)$ within RR, and the maximal flow rates $\left(Q_{\max , \text { sys }}\right.$ and $\left.Q_{\max , \text { dia }}\right)$ during the systolic, $R R_{s y s}$, and diastolic, $R R_{d i a}$, periods, respectively. $V_{\text {sys }}$ and $V_{d i a}$, identifying the blood volumes through the selected LAD section during $R R_{\text {sys }}$ and $R R_{\text {dia }}$, were defined as

$$
\begin{aligned}
& V_{s y s}=\int_{R R_{s y s}} Q_{L A D}(t) d t, \\
& V_{d i a}=\int_{R R_{d i a}} Q_{L A D}(t) d t .
\end{aligned}
$$

$V_{\text {sys }}$ and $V_{\text {dia }}$ enabled the calculation of the LAD stroke volume, $S V=V_{\text {sys }}+V_{d i a}$. From $S V$, the coronary blood flow, $C B F=S V * H R[\mathrm{ml} / \mathrm{min}]$ - which is a measure of the flow rate at coronary level - was finally assessed. Finally, the coronary perfusion pressure, $C P P$, was evaluated. It is the difference between the aortic diastolic pressure and the left-ventricular end-diastolic pressure.

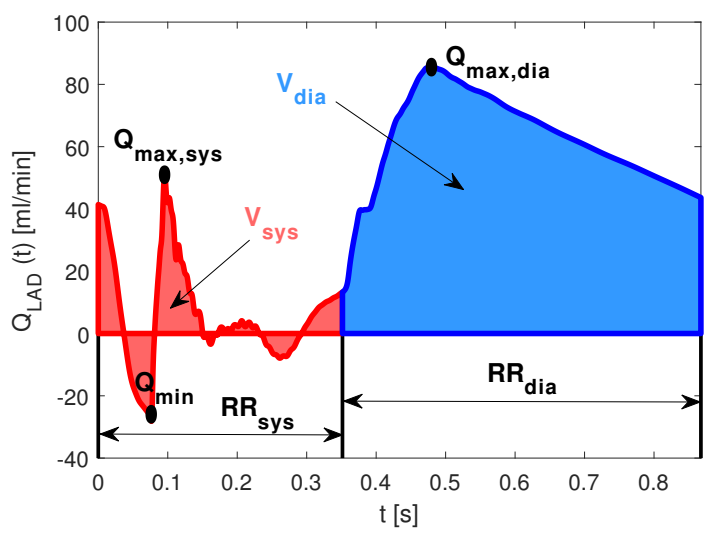

Figure 1. Example of fibrillated LAD flow rate waveform. $Q_{\max , s y s}$ and $Q_{\max , d i a}$ are the maximal flow rates during the systolic $R R_{s y s}$ and diastolic $R R_{\text {dia }}$ periods, $Q_{\min }$ is the minimum flow rate, $V_{\text {sys }}$ (red) and $V_{\text {dia }}$ (blue) are the blood volumes flowing through the chosen LAD section within $R R_{\text {sys }}$ and $R R_{d i a}$.

The mean values of $R R_{d i a}$ and $R R_{s y s}$ simulated at each $\mathrm{HR}$, together with the ratios between mean $R R_{d i a}$ and
$R R_{\text {sys }}$ for all frequencies, are reported in Tab.1. Here, one can note that mean $R R_{\text {sys }}$ and $R R_{d i a}$ become smaller increasing HR. Nevertheless, $R R_{\text {dia }}$ reduces faster than $R R_{\text {sys }}$ as HR rises. In fact, the ratio between mean $R R_{\text {dia }}$ and mean $R R_{\text {sys }}$ decreases with HR.

Table 1. (Columns II and III) Mean values \pm standard deviations and coefficients of variation (in brackets) of $R R_{\text {sys }}$ and $R R_{d i a}$ for each simulated frequency $(50,70$, $90,110,130 \mathrm{bpm}$ ). (Column IV) Ratio between mean val-

\begin{tabular}{|c|c|c|c|}
\hline $\begin{array}{c}\mathrm{HR} \\
{[\mathrm{bpm}]}\end{array}$ & $\begin{array}{c}R R_{\text {sys }} \\
{[\mathrm{s}]}\end{array}$ & $\begin{array}{c}R R_{\text {dia }} \\
{[\mathrm{s}]}\end{array}$ & 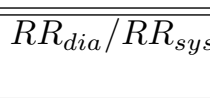 \\
\hline 50 & $\begin{array}{c}0.41 \pm 0.049 \\
(0.12)\end{array}$ & $\begin{array}{c}0.79 \pm 0.26 \\
(0.33)\end{array}$ & 1.93 \\
\hline 70 & $\begin{array}{c}0.35 \pm 0.034 \\
\quad(0.097)\end{array}$ & $\begin{array}{c}0.50 \pm 0.17 \\
(0.34)\end{array}$ & 1.43 \\
\hline 90 & $\begin{array}{c}0.32 \pm 0.026 \\
\quad(0.081)\end{array}$ & $\begin{array}{c}0.35 \pm 0.14 \\
(0.35)\end{array}$ & 1.09 \\
\hline 110 & $\begin{array}{c}0.31 \pm 0.019 \\
\quad(0.061)\end{array}$ & $\begin{array}{l}0.24 \pm 0.11 \\
\quad(0.32)\end{array}$ & 0.77 \\
\hline 130 & $\begin{array}{c}0.30 \pm 0.019 \\
(0.063)\end{array}$ & $\begin{array}{c}0.17 \pm 0.096 \\
\quad(0.56)\end{array}$ & 0.57 \\
\hline
\end{tabular}
ues of $R R_{d i a}$ and $R R_{\text {sys }}$.

\section{4. $\quad$ Results}

Tab.2 shows the mean values $(\mu)$, standard deviations $(\sigma)$ and coefficients of variation ( $c v)$ of $Q_{\max , s y s}$, $Q_{\max , d i a}$ and $Q_{\min }$ for each HR. From Tab.2, one can see that mean values of $Q_{\max , s y s}$ and $Q_{\max , d i a}$ increase moving towards the high frequency range, with the only exception of the mean value of $Q_{\max , \text { dia }}$ at $130 \mathrm{bpm}$. Standard deviations and coefficients of variation of $Q_{\max , s y s}$, $Q_{\text {max,dia }}$ rise with HR. However, the values of $\sigma$ and $c v$ for $Q_{\max , \text { sys }}$ slightly reduce at $130 \mathrm{bpm}$. This seems to suggest that the system tends to amplify diastolic fluctuations, damping systolic ones, at high frequency. Concerning $Q_{\min }$, the mean values, standard deviations and coefficients of variations remain almost constant within the whole frequency range. This means that $Q_{\min }$ is less sensitive than $Q_{\max , s y s}, Q_{\max , d i a}$ to $\mathrm{HR}$ variation during $\mathrm{AF}$.

The mean behaviours of $S V$ and $C B F$ are reported in Fig2. Fig.2a displays a reduction in $S V$ as cardiac frequency gets higher. This reduction is a direct consequence of lower $R R_{d i a}$ values when HR rises (Tab.1). In fact, keeping in mind the typical shape of the generic LAD flow rate signal (Fig.1), it is noticeable that the major contribution to $S V$ comes from $V_{d i a}$ (blue area). $V_{d i a}$ reduces at the higher frequencies because of the diminution in $R R_{\text {dia }}$. Thus, despite the growth in mean values of $V_{\text {sys }}$ with HR, there is no way to maintain constant $S V$ when moving towards the high frequency range. 

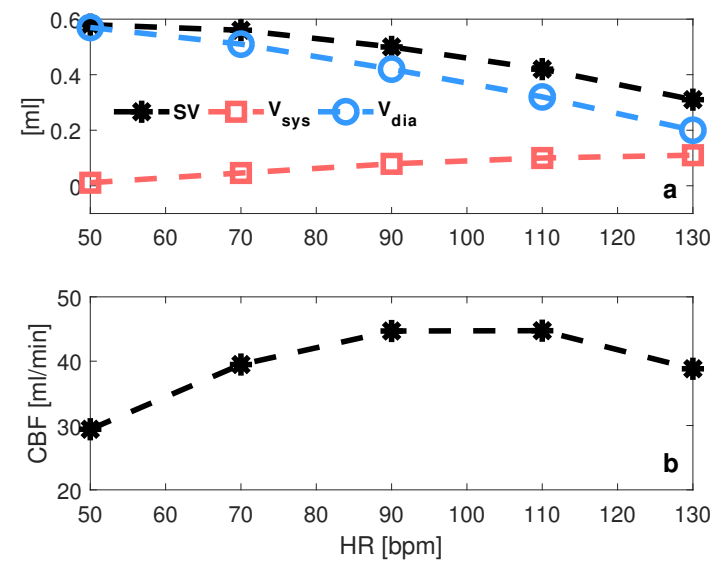

Figure 2. (a) Mean behaviours of $S V$ (LAD stroke volume), $V_{\text {sys }}$ (LAD blood volume during $R R_{\text {sys }}$ ), $V_{\text {dia }}$ (LAD blood volume during $R R_{d i a}$ ). (b) Dependance of $C B F$ (coronary blood flow) on HR.

Table 2. Mean values $(\mu)$ and standard deviations $(\sigma)$ of $Q_{\max , s y s}, Q_{\max , \text { dia }}$ and $Q_{\min }$ for each simulated cardiac frequency $(50,70,90,110,130 \mathrm{bpm})$ in AF. Coefficients of variation $(c v=\sigma / \mu)$ are indicated in brackets.

\begin{tabular}{cccc}
\hline \hline $\begin{array}{c}\mathrm{HR} \\
{[\mathrm{bpm}]}\end{array}$ & $\begin{array}{c}Q_{\max , \text { sys }} \\
{[\mathrm{ml} / \mathrm{min}]}\end{array}$ & $\begin{array}{c}Q_{\max , d i a} \\
{[\mathrm{ml} / \mathrm{min}]}\end{array}$ & $\begin{array}{c}Q_{\min } \\
{[\mathrm{ml} / \mathrm{min}]}\end{array}$ \\
\hline 50 & $48.97 \pm 5.36$ & $73.92 \pm 7.07$ & $-27.65 \pm 3.54$ \\
& $(0.11)$ & $(0.096)$ & $(-0.13)$ \\
70 & $56.36 \pm 10.65$ & $86.75 \pm 8.60$ & $-28.06 \pm 3.03$ \\
& $(0.19)$ & $(0.099)$ & $(-0.11)$ \\
90 & $71.11 \pm 15.07$ & $94.30 \pm 9.70$ & $-27.86 \pm 3.92$ \\
& $(0.21)$ & $(0.10)$ & $(-0.14)$ \\
110 & $84.68 \pm 15.08$ & $96.61 \pm 11.15$ & $-27.49 \pm 3.81$ \\
& $(0.18)$ & $(0.12)$ & $(-0.14)$ \\
130 & $87.41 \pm 14.85$ & $89.52 \pm 17.24$ & $-25.93 \pm 5.38$ \\
& $(0.17)$ & $(0.19)$ & $(-0.21)$ \\
\hline \hline
\end{tabular}

The mean behaviour of $C B F$ (Fig.2b) is not trivial. Since $C B F=S V * H R$ and mean $S V$ reduces with $\mathrm{HR}$, one could expect that mean $C B F$ also decreases from the lowest to the highest frequency. Instead, we found that mean $C B F$ rises from 50 to $90 \mathrm{bpm}$, remains almost constant between 90 and $110 \mathrm{bpm}$, finally decreasing for higher frequencies. According to this result, it appears that, up to $90 \mathrm{bpm}$, the reduction in $S V$ it is not sufficient to induce the impairment of the coronary flow perfusion.

$C P P$ is often used in literature as a surrogate measure for myocardial perfusion [7]. Thus, we are interested in verifying if it is possible to consider $C P P$ as a good estimate of $C B F$, even in AF. For this reason, beside the mean behaviours of $C B F$ with $\mathrm{HR}$, a focus on the $C B F$
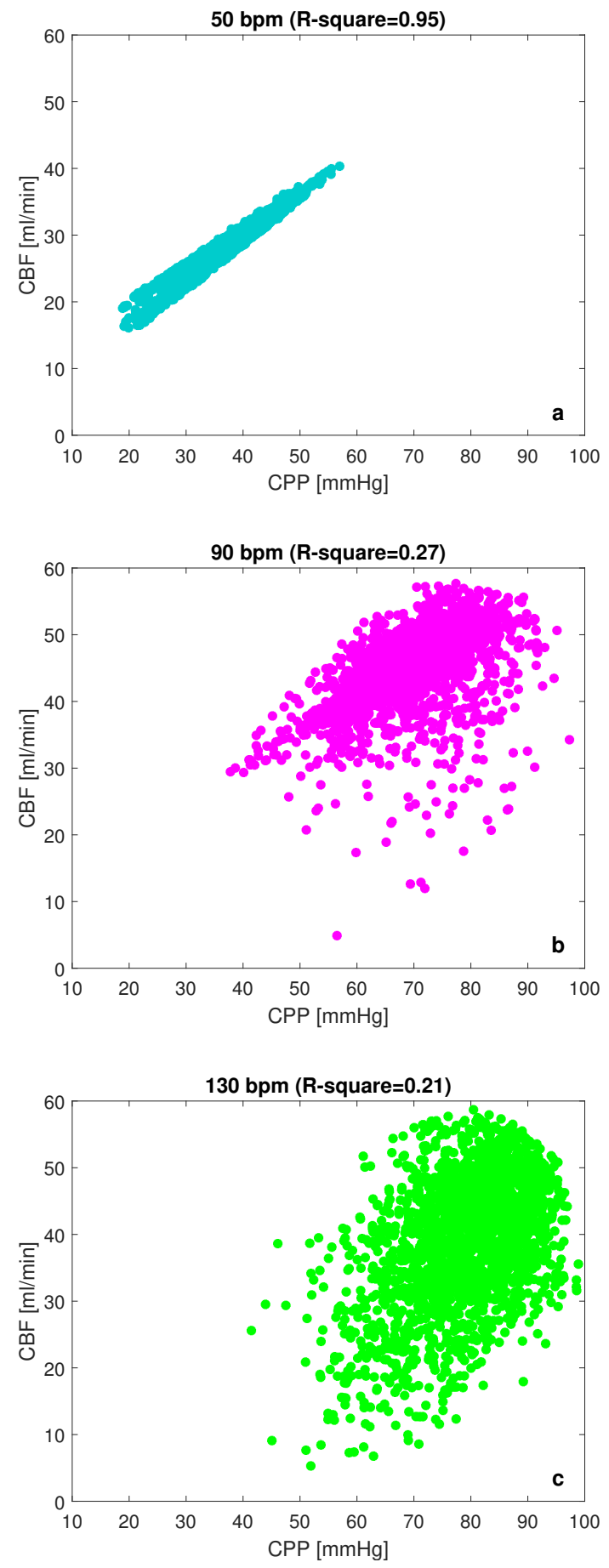

Figure 3. (a-c) Scatter plots between $C P P$ and $C B F$ at a LAD section for different HRs, with the corresponding R-square values.

data distribution with respect to $C P P$ was done at various frequencies in panels a-c of Fig.3. Fig.3 demonstrates that $C B F$ positively correlates with $C P P$ up to $70 \mathrm{bpm}$, but data become sparse and very low correlation is detectable 


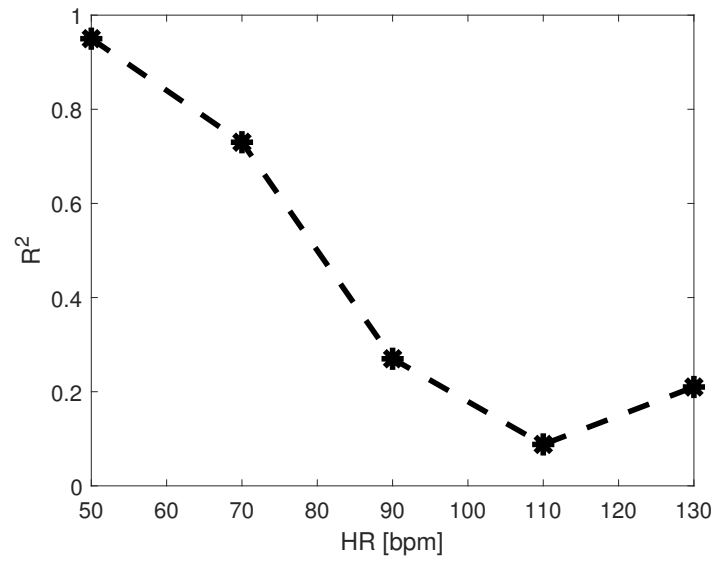

Figure 4. R-square behaviour of the scatter plots between $C B F$ and $C P P$ with $\mathrm{HR}$.

for higher HRs. In fact, as shown in Fig.4, there is a dramatic drop of the R-square $\left(R^{2}\right)$ values with HR: at the lowest HR, $R^{2}$ is close to one, while for $H R \geq 90 \mathrm{bpm}$ $R^{2}$ is damped under 0.3 . Moreover, the $R^{2}$ decrease with HR is not monotone, having the smallest $R^{2}$ value at 110 bpm rather than at $130 \mathrm{bpm}$.

In light of the presented results, it can be asserted that AF does not exclude correlation between $C B F$ and $C P P$, provided that cardiac frequencies remain small enough. When this condition is not respected, not only responses are irregular and not linear, but also the range of possible hemodynamic values enlarges. Evidence of this is given in panels a-c of Fig.3, where it is apparent that the amplitude of possible $C B F$ and $C P P$ values is remarkable at the higher frequencies, with respect to the smaller ones.

\section{Limitation}

The absence of a baroreceptor mechanism in the arterial model is a limiting aspect of the present work. In fact, we expect that in presence of a suitable barorecetor system, the decay in the LAD stoke volume would be attenuated, generating a maximum $C B F$ at smaller frequencies.

\section{Conclusion}

It is possible to summarize the three main outcomes of this study.

1) It emerges that a rise in the mean cardiac frequency during $\mathrm{AF}$, on average, leads to higher maximal flow rates during the systolic and diastolic periods but has an almost negligible effect on minimal flow rates (localised within the systolic periods), which remain almost unaffected. Moreover, fluctuations around mean values of maximal flow rates become more marked moving towards the high frequency range, with predominant systolic fluctuations up to $110 \mathrm{bpm}$ and a progressive amplification in the diastolic oscillations from 50 to $130 \mathrm{bpm}$.

2) Mean values of $S V$ decay with $\mathrm{HR}$, and $C B F$ presents a maximum value around $90-110 \mathrm{bpm}$. In other words, coronary perfusion begins to be impaired exceeding 90-110 bpm.

3) $C B F$ positively correlates with $C P P$ up to $70 \mathrm{bpm}$, but this correlation is lost at higher frequencies, for which data become sparse. Moreover, as HR is small enough $(50 / 70 \mathrm{bpm})$ linear responses are possible even in case of $\mathrm{AF}$, however, high frequencies (above $70 \mathrm{bpm}$ ) make disappear any form of linearity. Thus, based on previous results, $C P P$ cannot be considered a good estimate of $C B F$ for high HRs in AF.

\section{References}

[1] Guala A, Camporeale C, Tosello F, Canuto C, Ridolfi L. Modelling and subject-specific validation of the heartarterial tree system. Annals of Biomedical Engineering 2015;43:222-237.

[2] Kochiadakis GE, Kallergis EM. Impact of atrial fibrillation on coronary blood flow: a systematic review. Journal of Atrial Fibrillation 2012;5:458-462.

[3] Mynard JP, Penny DJ, Smolich JJ. Scalability and in vivo validation of a multiscale numerical model of the left coronary circulation. American Journal of Physiology-Heart and Circulatory Physiology 2014;306:H517-H528

[4] Scarsoglio S, Guala A, Camporeale C, Ridolfi L. Impact of atrial fibrillation on the cardiovascular system through a lumped.parameter approach. Medical \& Biological Engineering \& Computing 2014;52:905-920.

[5] Scarsoglio S, Gallo C, Ridolfi L. Effects of atrial fibrillation on the arterial fluid dynamics: a modelling perspective. Meccanica 2018;53(13):3251-3267.

[6] Zoni-Berisso M, Lercari F, Carazza T, Domenicucci S. Epidemiology of atrial fibrillation: European perspective. Clinical Epidemiology 2014;6:213-220.

[7] Westerhof N, Stergiopulos N, Noble MI. Snapshots of Hemodynamics: An Aid for Clinical Research and Graduate Education. New York: Springer, 2010.

Address for correspondence:

Caterina Gallo

DIMEAS, Politecnico di Torino, Torino 10129, ITALY

caterina.gallo@polito.it 